\title{
CXCR3 ligands are augmented during the pathogenesis of pulmonary sarcoidosis
}

\author{
A. Busuttil*, S.S. Weigt*, M.P. Keane ${ }^{\#}$, Y.Y. Xue*, V. Palchevskiy*, M.D. Burdick\%, \\ C. Huang*, D.A. Zisman*, M. Fishbein ${ }^{+}$, J.P. Lynch III*, R.M. Strieter", \\ R.M. Elashoff ${ }^{\S}$ and J.A. Belperio*
}

ABSTRACT: We and other investigators have hypothesised that the CXC chemokine receptor (CXCR) $3 / C X C R 3$ ligand biological axis is involved in the formation of sarcoid lung granulomas; however, significant discrepancies in the current literature remain. In an effort to clarify previous conflicting findings, we performed the largest observational study to date of interferon-inducible ELR $^{-}$(lacking the sequence glutamic acid-leucine-arginine) CXC chemokines in sarcoid bronchoalveolar fluid (BALF).

BALF chemokine levels from sarcoid patients $(n=72)$ and healthy controls $(n=8)$ were measured with the ELISA method. Immunohistochemical staining was performed for CXCR3 and its ligands.

BALF CXC chemokine ligand (CXCL) 10 levels from sarcoid patients were not significantly increased compared with controls. BALF CXCL11 levels from sarcoid patients demonstrated a trend towards elevation; subgroup analysis by stage showed significant BALF CXCL11 elevation in stage I sarcoid patients compared with controls. BALF CXCL9 levels were elevated from sarcoid patients compared with controls. CXC11, CXCL9 and CXCR3 were expressed from epithelioid histiocytes, multinucleated giant cells and other inflammatory cells forming sarcoid lung granulomas.

Our data suggest that CXCL9 and CXCL11 are important mediators in recruiting CXCR3expressing cells. Importantly, we have made the novel observation that both lymphocytes and cells of monocyte linage express CXCR3 and are involved in the formation of sarcoid lung granulomas.

KEYWORDS: Chemokines, chemokine receptors, leukocytes, lung, sarcoidosis

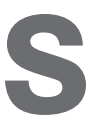
arcoidosis is a systemic inflammatory disease of unknown aetiology. In severe cases, sarcoidosis can progress to endstage lung disease, necessitating lung transplantation or resulting in death [1]. The diagnosis of pulmonary sarcoidosis is established when clinical and radiographical findings are supported by the histological features of discrete, well-formed, non-necrotising granulomas in the absence of infectious diseases or inorganic material [2]. Although multiple organ systems can be affected, lung involvement predominates [2-4]. During the pathogenesis of pulmonary sarcoidosis there is typically an early cellular precursor lesion, which causes an inflammatory milieu that directs the recruitment of epithelioid mononuclear phagocytes and the eventual formation of multinucleated giant cells [5]. This ultimately results in the classic non-necrotising granuloma of pulmonary sarcoidosis [5].
Chemokines are small proteins that are important for the recruitment of leukocytes. The CXC chemokine subfamily is divided by the presence or absence of the sequence glutamic acidleucine-arginine (ELR) near the $\mathrm{NH}_{2}$-terminal. $\mathrm{ELR}^{+} \mathrm{CXC}$ chemokines are neutrophil chemoattractants with angiogenic properties [6-8]. The ELR $^{-}$CXC chemokines are lymphocyte chemoattractants with angiostatic properties [6-8]. The ELR $^{-}$CXC chemokines can be further subclassified into interferon (IFN)-inducible and non-IFN-inducible ELR ${ }^{-}$CXC chemokines [6-8]. The IFNinducible ELR ${ }^{-}$CXC chemokines are known for their potent chemoattraction for type 1 mononuclear cells and mediate the recruitment of these cells through the $\mathrm{G}$ protein-coupled cell surface receptor CXC chemokine receptor (CXCR)3 [6-8]. Given the mononuclear cell predominance in characteristic sarcoid lesions, we hypothesised that IFN-inducible ELR ${ }^{-}$CXC chemokines (monokine

\section{AFFILIATIONS}

*Division of Pulmonary and Critical Care Medicine, Dept of Medicine, and +Dept of Pathology and Laboratory Medicine, David Geffen School of Medicine at UCLA, Los Angeles, CA, ${ }^{\S}$ Dept of Biomathematics, University of California, Los Angeles, CA, and -Division of Pulmonary and Critical Care Medicine, Dept of Medicine, University of Virginia Health System, Charlottesville, VA, USA.

\# Dept of Medicine, St Vincent's University Hospital and University College Dublin, Dublin, Ireland.

\section{CORRESPONDENCE}

J.A. Belperio

Dept of Medicine, Division of Pulmonary and Critical Care Medicine David Geffen School of Medicine at UCLA

Room 14-154 Warren Hall

Box 711922

900 Veteran Ave.

Los Angeles

CA 90095-1786

USA

E-mail: jbelperio@mednet.ucla.edu

Received:

Oct 172008

Accepted after revision:

March 282009

First published online:

April 222009 
induced by IFN- $\gamma$ (MIG)/CXC chemokine ligand (CXCL)9, IFN- $\gamma$-inducible protein (IP)-10/CXCL10 and IFN-inducible Tcell $\alpha$ chemoattractant (ITAC)/CXCL11) would be involved in the pathogenesis of sarcoidosis.

This study was designed to determine whether there are augmented levels of IFN-inducible ELR ${ }^{-}$CXC chemokines in bronchoalveolar lavage fluid (BALF) from patients with the clinical/pathological diagnosis of pulmonary sarcoidosis, and resolve the current discrepancies within the literature [9-13]. Moreover, this study aimed to elucidate the cellular sources of specific IFN-inducible ELR- CXC chemokines and determine which cells express CXCR3 during the pathogenesis of pulmonary sarcoidosis.

\section{MATERIALS AND METHODS}

\section{Study design and patient population}

With Institutional Review Board approval (David Geffen School of Medicine at UCLA, Los Angeles, CA, USA) and informed written consent, we prospectively enrolled all patients undergoing bronchoscopy for interstitial lung disease from June 1993 to December 2003. Patients were eligible for enrolment if they were suspected of having an interstitial lung disease. For this specific study we retrospectively identified 72 patients who had an initial de novo confirmed clinical/ pathological diagnosis of sarcoidosis. All patients underwent a bronchoscopy with transbronchial lung biopsy with or without a video-assisted thoracoscopic (VATS) biopsy, open lung biopsy or mediastinoscopy. The diagnosis of sarcoidosis was made if the patient's lung biopsy (transbronchial biopsy $(n=59,82 \%)$ and surgical biopsy $(n=13,18 \%))$ showed discrete, well-formed, non-necrotising granulomas without evidence of infection or inorganic material to account for the pulmonary granulomatous reaction. Throughout the entire study we excluded any bronchoalveolar lavage (BAL) performed at a time when infection and/or colonisation was diagnosed via the following criteria: BALF or lung biopsy positive Gram stain or culture for bacteria, acid-fast bacillus, cytomegalovirus, respiratory viruses, Pneumocystis jiroveci or other fungal organisms. Additionally, patients with positive histoplasmosis serum serology or urine antigen or positive coccidioidimycoses enzyme immunoassay confirmed with a positive complement fixation were excluded.

All patients with sarcoidosis were staged retrospectively using a modification of the system outlined by the American Thoracic Society (ATS)/European Respiratory Society (ERS)/World Association of Sarcoidosis and Other Granulomatous Disorders joint statement as previously described [2, 14]. Criteria were as follows. Stage I: bilateral hilar lymphadenopathy without pulmonary infiltrates; stage II: bilateral hilar lymphadenopathy with pulmonary infiltrates; and stage III: pulmonary infiltrates and/or fibrosis in the absence of hilar lymphadenopathy $[2,14]$. In addition, we also retrospectively characterised these patients with or without an active pulmonary lymphocytic alveolitis defined by a BAL lymphocyte count $>30 \times 10^{3}$ lymphocytes $\cdot \mathrm{mL}^{-1}$ as previously described [9]. Furthermore, we identified eight sarcoid patients being treated with empiric methylprednisolone therapy for symptoms of arthralga at the time of their diagnostic bronchoscopy (table 1). BALF was also obtained from eight healthy control patients without any past medical history, pulmonary symptoms or abnormal radiographical findings.

\section{BALF collection for chemokine analysis}

BALF was obtained from patients with sarcoidosis and from normal healthy controls by methods previously described [15-17]. Briefly, a flexible bronchoscope was wedged into a subsegmental bronchus of a predetermined region of interest based on radiographical findings. A BAL was performed by instilling a total of $240 \mathrm{~mL}$ of normal saline in $60-\mathrm{mL}$ aliquots, each retrieved by low suction. The BALF fractions were pooled and split equally into two samples. One sample was sent to the clinical microbiology and cytology laboratory and the other sample was placed on ice and transported to the research laboratory. The research sample was filtered through a sterile gauze and centrifuged for $10 \mathrm{~min}$ at $500 \times g$. The cell-free solution was aliquoted and frozen immediately at $-70^{\circ} \mathrm{C}$ until thawed for chemokine ELISAs.

\section{Reagents}

Mouse antibody to human CXCL9 and goat antibody to human CXCR3 were purchased from R\&D Systems (Minneapolis, MN, USA). Mouse antibody to human CXCL11 was purchased from Santa Cruz Biotechnology Inc. (Santa Cruz, CA, USA). Mouse antibody to human CD68 was purchased from DakoCytomation (Carpinteria, CA, USA).

\section{CXCL9, CXCL10 and CXCL11 chemokine ELISAs}

Human BALF protein levels of CXCL9, CXCL10 and CXCL11 were quantitated according to the manufacturer's protocol

\begin{tabular}{lccc}
\hline TABLE 1 & Pulmonary sarcoidosis patient characteristics \\
Patient characteristics & Stage I & Stage II & Stage III \\
\hline & & & \\
Patients n & 27 & 36 & 9 \\
Age yrs & $39 \pm 8$ & $39 \pm 11$ & $54 \pm 10$ \\
Sex & & & \\
$\quad$ Female & $10(37)$ & $21(58)$ & $7(78)$ \\
$\quad$ Male & $17(63)$ & $15(42)$ & $2(22)$ \\
Race & & & \\
$\quad$ Black & $9(34)$ & $12(34)$ & $3(33)$ \\
White & $12(44)$ & $20(55)$ & $5(55)$ \\
$\quad$ Other & $6(22)$ & $4(11)$ & $1(12)$ \\
Lymphocytic alveolitis & $13(48)$ & $12(33)$ & $2(22)$ \\
Immunosuppressive therapy & $5(18)$ & $3(8.3)$ & $0(0.0)$ \\
FEV 1 L & $3.05 \pm 0.86$ & $2.93 \pm 0.96$ & $1.50 \pm 0.62$ \\
FEV 1 \% pred & $87.58 \pm 21.21$ & $82.82 \pm 21.37$ & $61.67 \pm 24.99$ \\
FVC L & $3.77 \pm 1.25$ & $3.67 \pm 1.05$ & $2.41 \pm 1.10$ \\
FVC \% pred & $85.57 \pm 21.25$ & $83.52 \pm 18.89$ & $73.67 \pm 33.31$ \\
TLC L & $5.94 \pm 0.93$ & $5.49 \pm 1.13$ & $3.25 \pm 0.00$ \\
TLC \% pred & $84.40 \pm 18.87$ & $83.78 \pm 37.75$ & $71.00 \pm 0.000$ \\
DL,Co mL·min ${ }^{-1} \cdot$ mmHg $^{-1}$ & $27.1 \pm 6.88$ & $23.2 \pm 7.43$ & $13.5 \pm 2.12$ \\
DL,Co \% pred & $90.62 \pm 17.21$ & $70.20 \pm 16.94$ & $61.00 \pm 8.485$ \\
& & &
\end{tabular}

Data are presented as mean \pm SD or $n(\%)$, unless otherwise stated. FEV 1 forced expiratory volume in $1 \mathrm{~s}$; \% pred: \% predicted; FVC: forced vita capacity; TLC: total lung capacity; DL,CO: diffusing capacity of the lung for carbon monoxide 

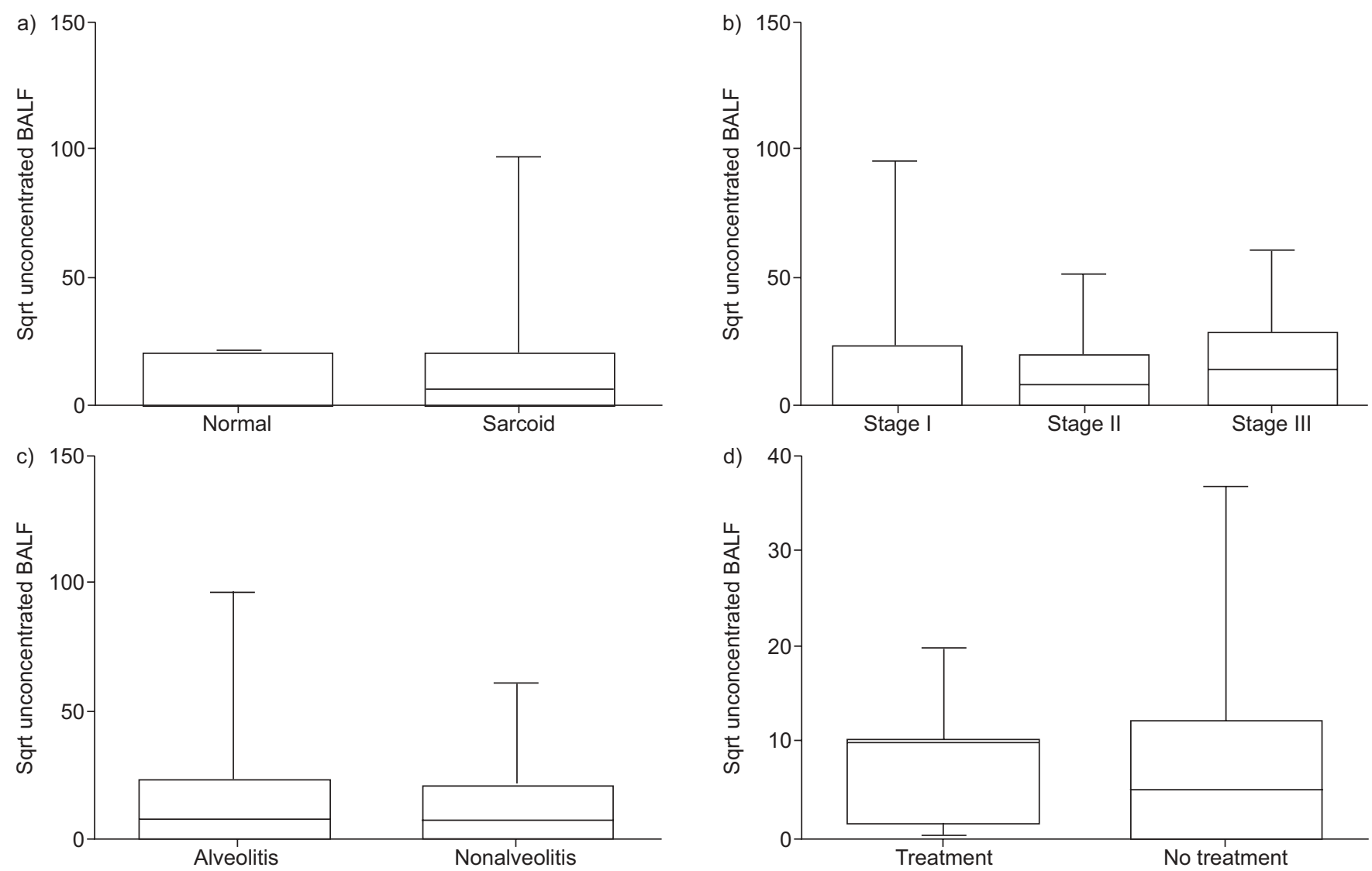

FIGURE 1. Bronchoalveolar lavage fluid (BALF) protein levels of CXC chemokine ligand (CXCL)10 from patients with sarcoidosis were not elevated compared with normal healthy controls. BALF protein levels of CXCL10 by ELISA from a) all sarcoidosis patients $(n=72)$ compared with normal healthy volunteers ( $n=8), b)$ different stages of sarcoidosis (stage I $(n=27)$, stage II $(n=36)$ and stage III $(n=9))$, c) sarcoid patients with $(n=27)$ and without $(n=45)$ alveolitis, and d) sarcoidosis patients with $(n=8)$ and without $(n=64)$ empirical treatment at the time of diagnosis. Sqrt: square root transformed.

using ELISA kits (R\&D Systems). Human CXCL9, CXCL10 and CXCL11 had the lowest detectable limit of 15, 12 and $17 \mathrm{pg} \cdot \mathrm{mL}^{-1}$ respectively.

\section{Immunohistochemistry for CXCR3 ligands, CXCR3 and CD68}

Immunohistochemistry was performed on paraffin-embedded slides of lung tissue from sarcoidosis patients for the localisation of the IFN-inducible ELR ${ }^{-}$CXC chemokines and their shared receptor, CXCR3, as previously described [15]. Briefly, after deparaffinisation and steam bath antigen retrieval in citrate buffer ( $\mathrm{pH} 6.0)$, endogenous perioxidase was quenched with 3\% hydrogen peroxide in 50\% methanol for $15 \mathrm{~min}$. Nonspecific binding was minimised by incubation in either 3\% normal rabbit serum (for anti-CXCR3) or 3\% normal horse serum (for all other antibodies) for $30 \mathrm{~min}$. Endogenous biotin was blocked with an avidin/biotin blocking kit according to the manufacturer's specifications (Vector Laboratories, Inc., Burlingame, CA, USA). Slides were then incubated overnight at $4^{\circ} \mathrm{C}$ with murine anti-human CXCL9 antibody (R\&D Systems), goat anti-human CXCR3 antibody (R\&D Systems), murine anti-human CXCL11 antibody (Santa Cruz), murine anti-human CD68 (DakoCytomation), or the appropriate isotype control immunoglobulin (Ig)G. Specific labelling was detected with a biotin-conjugated rabbit anti-goat or horse anti-murine secondary antibody and application of horseradish peroxidase-bound avidin/biotin from Vectastain $A B C$ kits (Vector Laboratories), followed by development with 3,3'diaminobenzidine (DAB) solution (Vector Laboratories).

\section{Statistical analysis}

Since all BALF chemokine protein levels had a skewed distribution, all analyses were performed on square root transformed data. Based on our overall hypothesis that the CXCR3/CXCR3 ligand biological axis is involved in the formation of non-necrotising granulomas in pulmonary sarcoidosis, our primary end-point was to determine whether there are augmented BALF protein levels of specific chemokines from patients with pulmonary sarcoidosis compared with healthy volunteers. For CXCL10 and CXCL11 we used the MannWhitney test. For CXCL9 we used the Fisher's exact test because multiple CXCL9 protein levels in our control samples were below the lower limit of assay detection. Previous chemokine studies involving sarcoidosis patients have performed multiple subgroup analyses. Thus, we performed exploratory end-points that evaluated differences in BALF chemokine levels using pairwise comparisons between patients with and without sarcoid alveolitis and patients with or without empiric immunosuppressive treatment at the time of sarcoid diagnosis via the Mann-Whitney test. Furthermore, we performed other exploratory analyses 

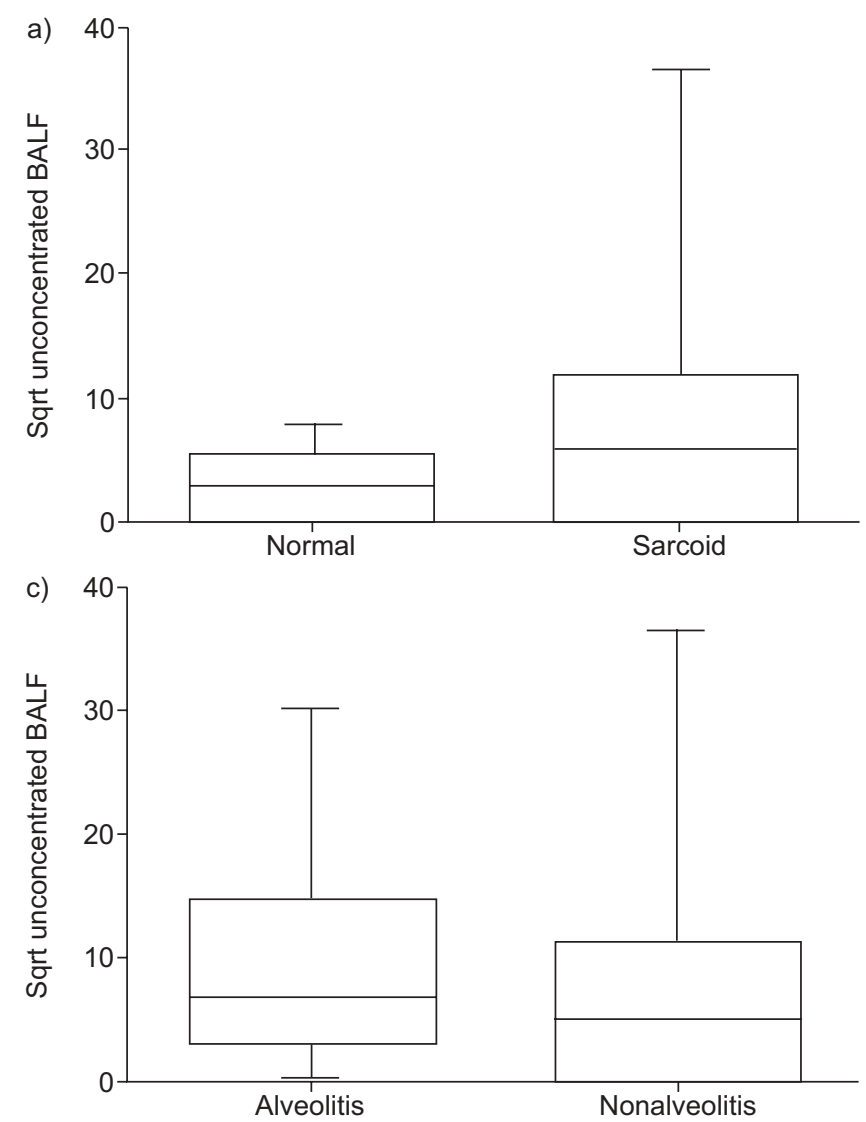

b)

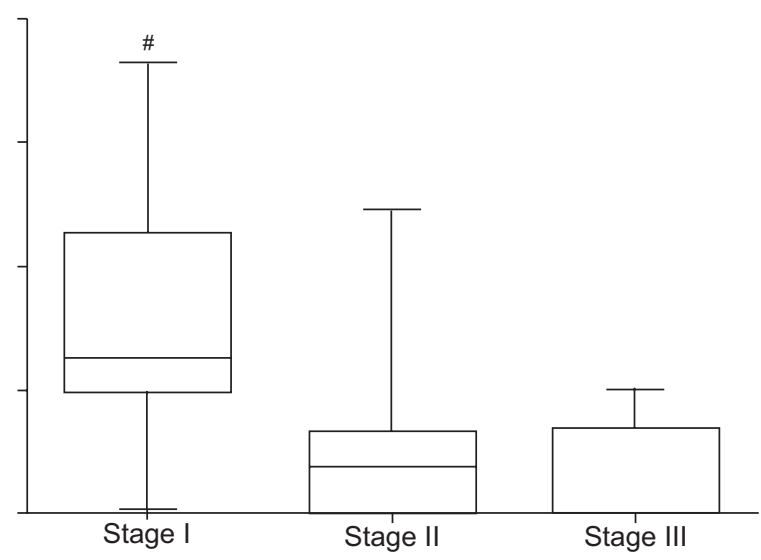

d)

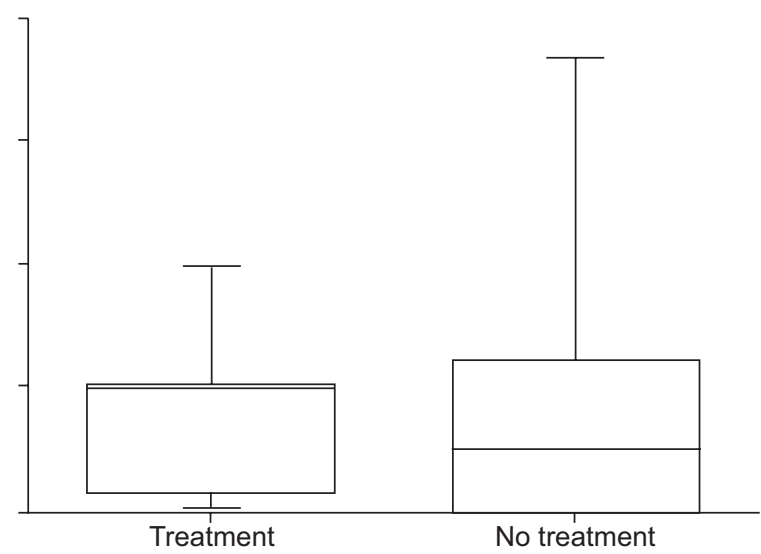

FIGURE 2. Bronchoalveolar lavage fluid (BALF) protein levels of CXC chemokine ligand (CXCL)11 from patients with stage I sarcoidosis were elevated. BALF protein levels of CXCL11 by ELISA from a) all sarcoidosis patients $(n=72)$ compared with normal healthy volunteers $(n=8), b)$ different stages of sarcoidosis (stage I $(n=27)$, stage II $(n=36)$ and stage III $(n=9))$, c) sarcoid patients with $(n=27)$ and without $(n=45)$ alveolitis, and d) sarcoidosis patients with $(n=8)$ and without $(n=64)$ empirical treatment at the time of diagnosis. Sqrt: square root transformed. ${ }^{*}: p=0.027$ for stage I versus stage III.

involving multigroup comparisons between BALF chemokines from patients with different stages of sarcoidosis using the Kruskal-Wallace test with post hoc Dunn. Additionally, exploratory comparisons were made between the various stages of sarcoidosis compared with healthy controls using the MannWhitney test for CXCL10 and CXCL11, and the Fisher's exact test for CXCL9. Data were displayed using a box plot summary. The plot's horizontal line represents the median, the box encompasses the 25-75th percentiles, and the error bars encompass the range of data points.

\section{RESULTS}

\section{Patient population}

Based on a modification of the ATS/ERS/World Association of Sarcoidosis and Other Granulomatous Disorders joint statement staging criteria [2, 14] and excluding patients with infection/colonisation, we identified $27(38 \%)$ patients with stage I disease, $36(50 \%)$ with stage II disease and nine $(12 \%)$ with stage III disease (table 1). Using the definition of active pulmonary lymphocytic alveolitis as a BAL lymphocyte count $>30 \times 10^{3}$ lymphocytes $\cdot \mathrm{mL}^{-1}$, there were $27(37 \%)$ patients with and $45(63 \%)$ patients without an active alveolitis (table 1). Eight $(11 \%)$ patients were on and $64(89 \%)$ patients were off empiric immunosuppressive treatment at the time of their diagnostic bronchoscopy (table 1). Additional sarcoidosis patient characteristics such as age, sex, race and pulmonary function testing are outlined in table 1. Eight healthy volunteers without pulmonary symptoms, with normal chest radiographs and no past medical history underwent a bronchoscopy and were deemed the healthy control group.

\section{CXCL10 protein levels in BALF from pulmonary sarcoidosis patients were not elevated}

BALF CXCL10 protein levels measured by ELISA were not significantly elevated in patients with sarcoidosis $(n=72)$ compared with normal healthy controls $(n=8)$ (fig. 1a). There were no significant differences in BALF CXCL10 levels comparing healthy patients with patients with stages I, II or III sarcoidosis. There were no significant differences in BALF CXCL10 levels in a three-group comparison across the different stages of sarcoidosis (stage I $(n=27)$, stage II $(n=36)$ and stage III $(n=9))$ (fig. 1b). The presence of a lymphocytic alveolitis had no impact on CXCL10 levels (fig. 1c). Furthermore, empiric immunosuppressive therapy at the time of diagnostic bronchoscopy did not affect CXCL10 levels (fig. 1d).

\section{CXCL11 protein levels in BALF from stage I pulmonary sarcoidosis patients were elevated}

BALF CXCL11 protein levels had a nonsignificant trend towards elevation in sarcoid patients compared with normal 

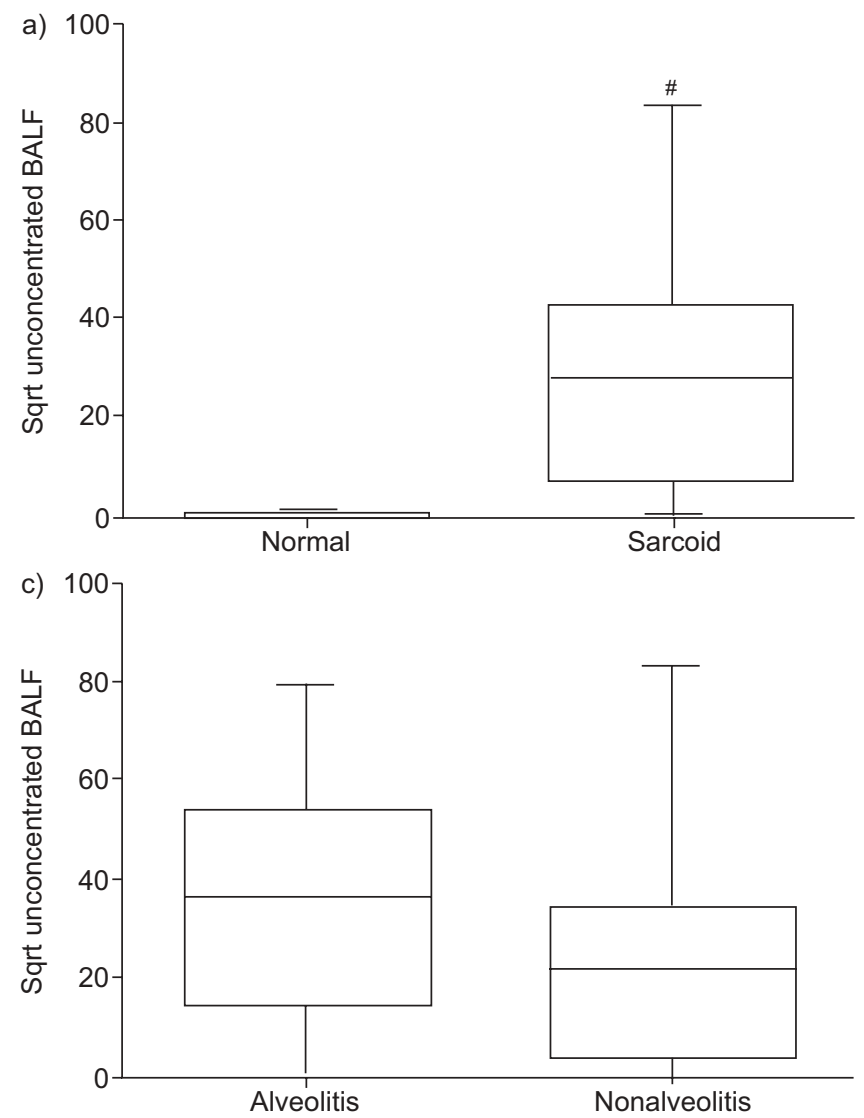

b)

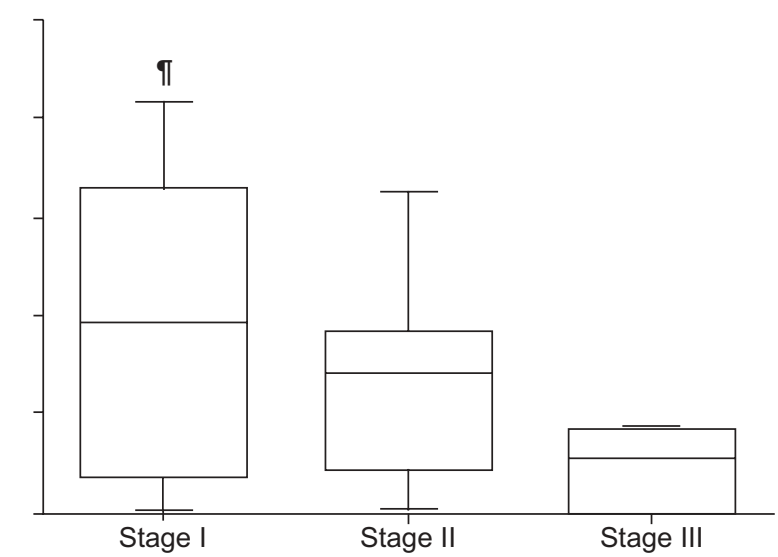

d)

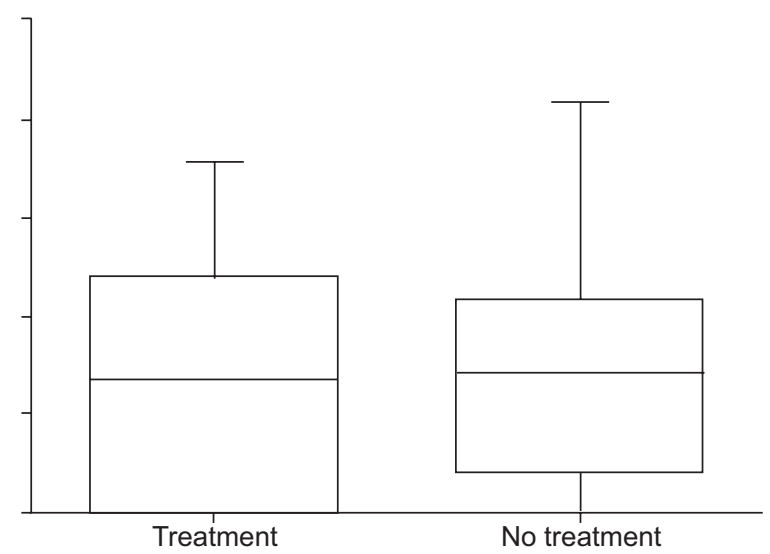

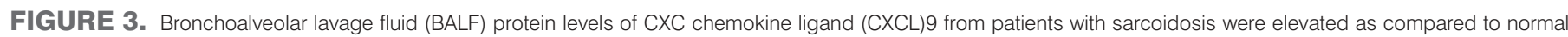

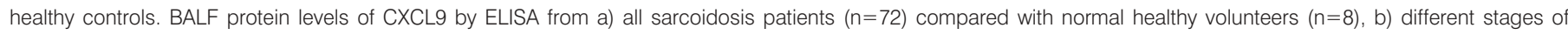

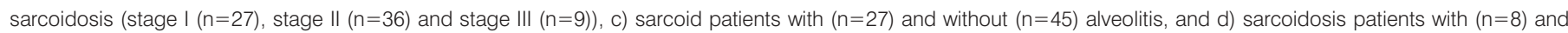

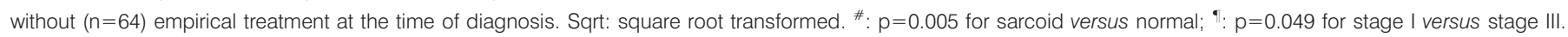

healthy controls $(p=0.064$; fig. 2a). However, BALF CXCL11 levels were elevated in patients with stage I sarcoidosis compared with healthy controls $(\mathrm{p}=0.001)$. In a three-group comparison across sarcoid stages (I, II and III), BALF CXCL11 protein levels varied significantly (fig. $2 b$ ). Specifically, stage I had a nonsignificant trend towards elevation compared with stage II $(p=0.073)$, and stage I was significantly elevated compared with stage III ( $p=0.027$; fig. $2 b)$. The presence of a lymphocytic alveolitis had no impact on CXCL11 levels (fig. 2c). Furthermore, empiric immunosuppressive therapy at the time of diagnostic bronchoscopy did not affect CXCL11 levels (fig. 2d).

\section{CXCL9 protein levels in BALF from pulmonary sarcoidosis patients were elevated}

BALF CXCL9 protein levels were significantly elevated from sarcoid patients compared with healthy controls $(p=0.005$; fig. 3a). Additionally, CXCL9 levels were elevated from patients with stages I and II sarcoidosis compared with healthy controls ( $\mathrm{p}=0.005$ and $\mathrm{p}=0.009$, respectively). BALF CXCL9 protein levels also varied significantly across the different stages (I, II and III) of sarcoid (fig. 3b). Specifically, stage II had a nonsignificant trend towards elevation compared with stage III $(p=0.059)$ and stage I was significantly elevated compared with stage III ( $p=0.049$; fig. $3 b)$. The presence of a lymphocytic alveolitis had no impact on CXCL9 levels (fig. 3c). Furthermore, empiric immunosuppressive therapy at the time of diagnostic bronchoscopy did not affect CXCL9 levels (fig. 3d).

\section{CXCR3 and its ligands CXCL9 and CXCL11 were localised to infiltrating mononuclear cells that form the granulomatous lesions of pulmonary sarcoidosis}

Stage I sarcoid specimens with and without alveolitis demonstrated a marked CXCL11 protein expression from epithelioid histiocytes and multinucleated giant cells forming nonnecrotising granulomas as well as from surrounding alveolar macrophages (fig. 4). Both stages I and II sarcoid specimens with and without alveolitis demonstrated a marked CXCL9 protein expression from epithelioid histiocytes within granulomas and less intense, albeit positive expression, from multinucleated giant cells and surrounding alveolar macrophages (fig. 5). Both stages I and II sarcoid specimens with and without alveolitis demonstrated marked CXCR3 protein expression from epithelioid histiocytes and multinucleated giant cells within the granulomatous formations, as well as from other infiltrating interstitial mononuclear cells (fig. 6). Using the monocyte linage marker CD68, we confirmed CXCR3 expression was from both epithelioid histiocytes and multinucleated giant cells (fig. 7). 
Anti-CXCL11
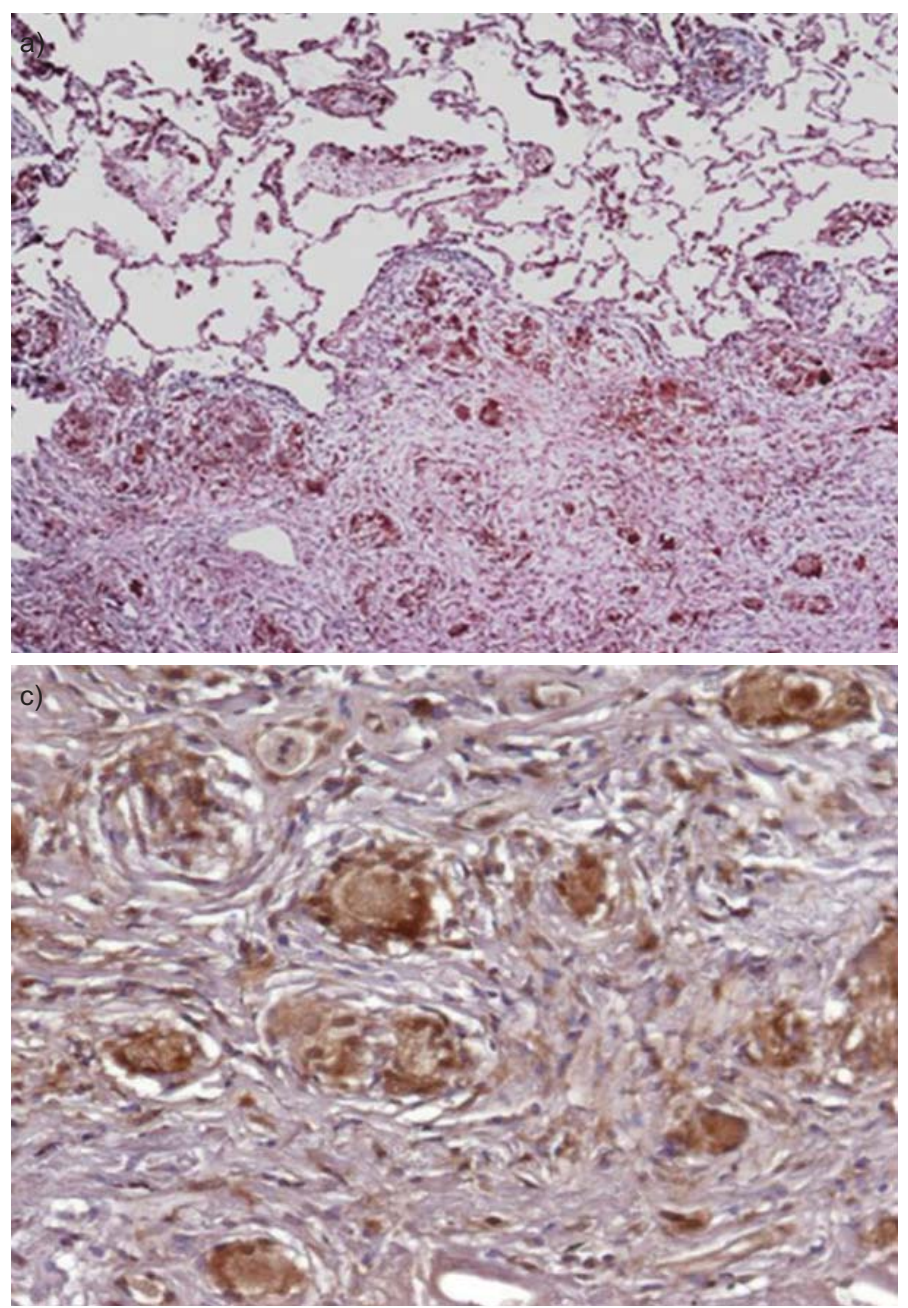

Control
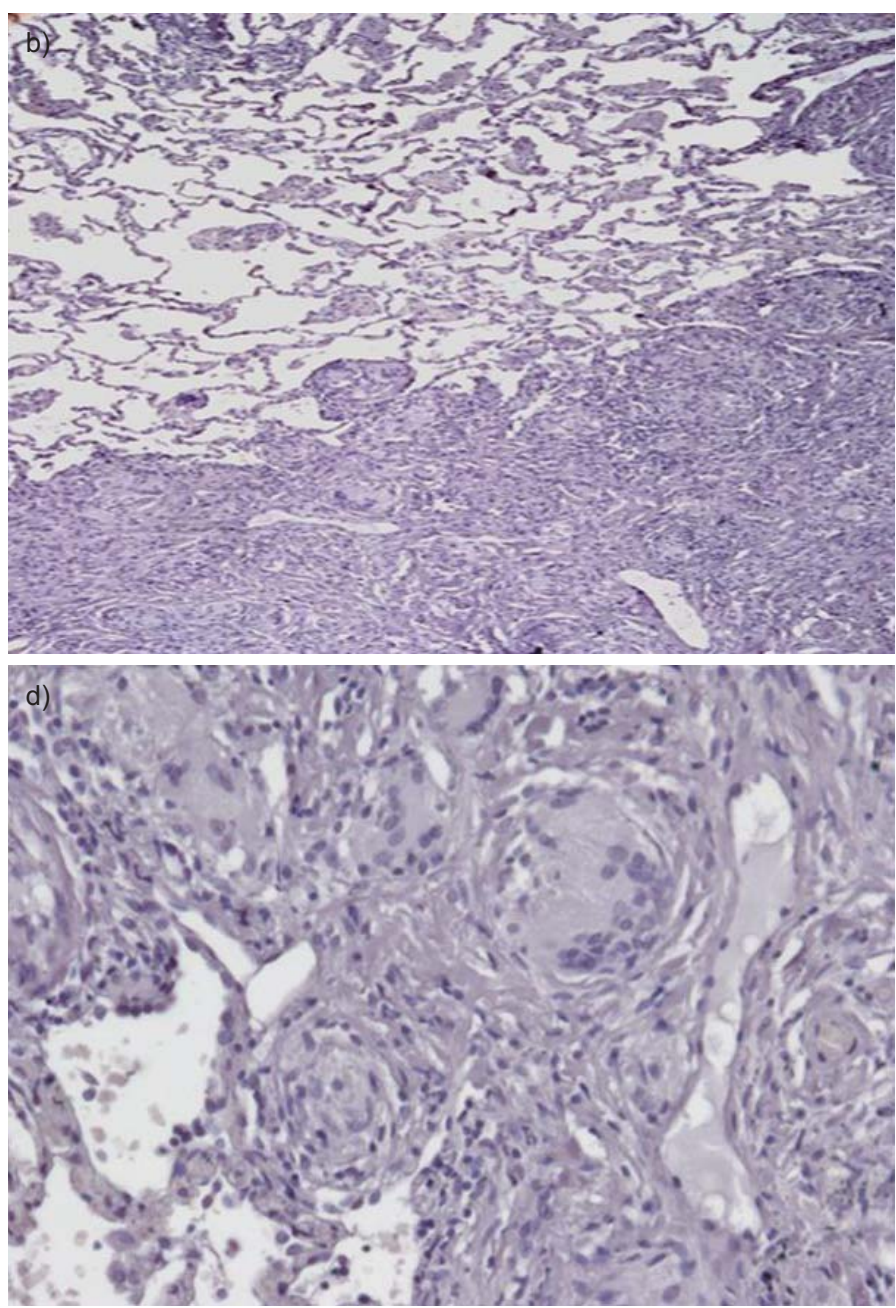

FIGURE 4. Representative immunohistochemistry for a and c) CXC chemokine ligand (CXCL)11 and b and d) isotype control antibody on lung biopsy tissue from stage I sarcoidosis. CXCL11 protein expression was found in epithelioid histiocytes and multinucleated giant cells forming non-necrotising granulomas, as well as from surrounding alveolar macrophages. Panels were photographed at $a$ and b) $50 \times$ and $c$ and d) $200 \times$ original magnification.

\section{DISCUSSION}

Previous studies involving BAL cells and fluid from sarcoid patients have demonstrated a type 1 immune response milieu with increased expression of tumour necrosis factor (TNF)- $\alpha$, interleukin (IL)-12, IL-18, and IFN- $\gamma$ [18-23]. Although an area of active investigation, the type 1 cytokine-chemokine network involved in the recruitment of mononuclear cells to the lungs of sarcoid patients has not been fully elucidated. We and other investigators have hypothesised that the CXCR3/CXCR3 ligand biological axis is involved in the formation of sarcoid lung granulomas; however, significant discrepancies in the current literature remain [9-13]. In an effort to clarify previous conflicting findings, we performed the largest observational study to date of IFN-inducible ELR ${ }^{-}$CXC chemokines in sarcoid BALF. Furthermore, we have elucidated the cellular sources of CXCR3 ligands and CXCR3 expression, a finding that helps clarify the cellular mechanism(s) of granuloma formation in pulmonary sarcoidosis.

Prior studies investigating CXCL10 in pulmonary sarcoidosis have shown conflicting results [9-11]. Specifically, AGostini et al.
[9] found increased levels of CXCL10 in BALF from patients with active sarcoidosis (defined as a lymphocytosis $>30 \times 10^{3}$ lymphocytes $\cdot \mathrm{mL}^{-1}$, a ratio of $\mathrm{CD} 4 / \mathrm{CD} 8$ T-cells $>5$ and a positive pulmonary gallium scan) $(n=17)$ compared with patients with inactive pulmonary sarcoidosis $(n=7)$ and normal controls $(n=6)$. These investigators found that most sarcoid patients had detectable levels of CXCL10 compared with virtually undetectable levels from their healthy controls [9]. SUGIYAMA et al. [10] also found elevated BALF levels of CXCL10 from patients with active sarcoidosis $(n=41)$ compared with volunteers $(n=12)$, despite all sarcoid patients being on immunosuppressive therapy. Conversely, ANTONIOU et al. [11] found significantly lower BALF levels of CXCL10 from patients with sarcoidosis $(n=20)$ compared with controls $(n=12)$. In the present study, we analysed a larger sarcoid population $(n=72)$ and did not find elevations in CXCL10 from BALF. We also did not find differences among different stages of sarcoid, those with or without alveolitis, or those on or off empiric immunosuppressive therapy at the time of sarcoid diagnosis. Interestingly, the levels of CXCL10 in our healthy controls were higher than those found by AGOSTIN et al. [9], which may be 

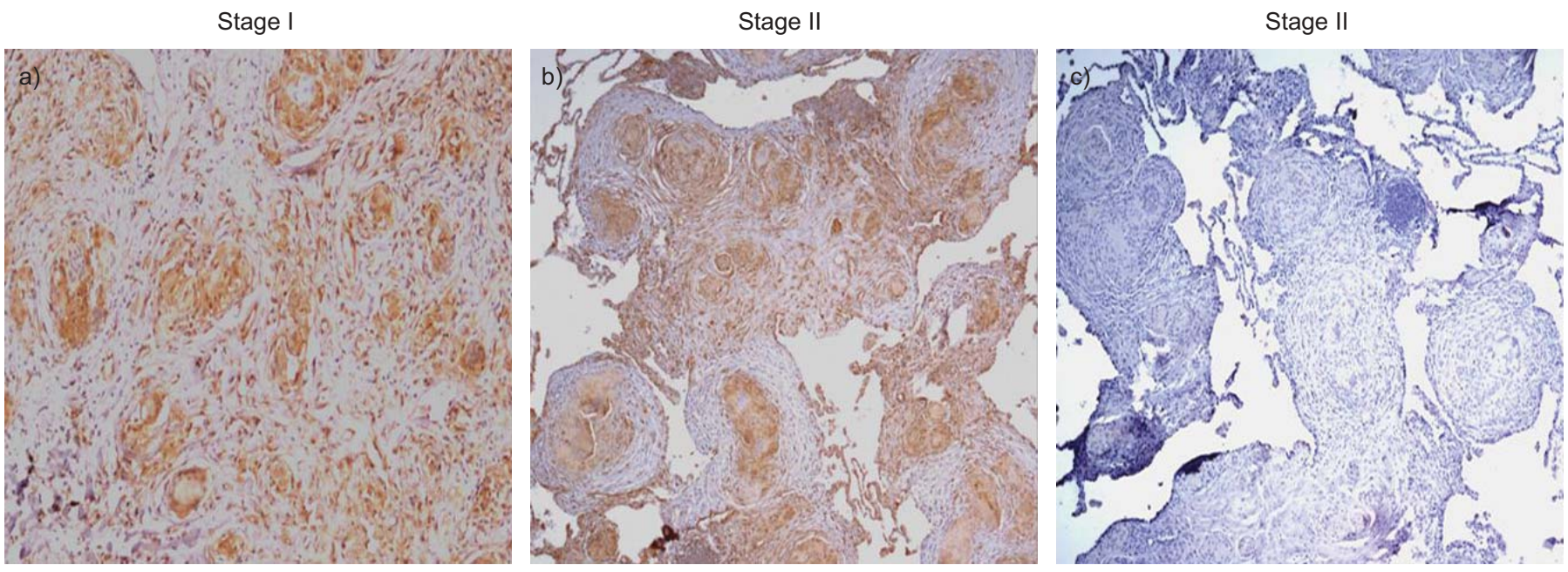

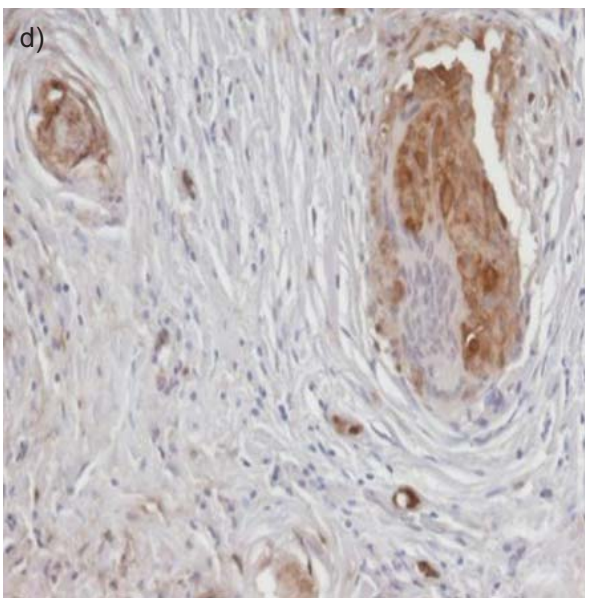

Anti-CXCL9

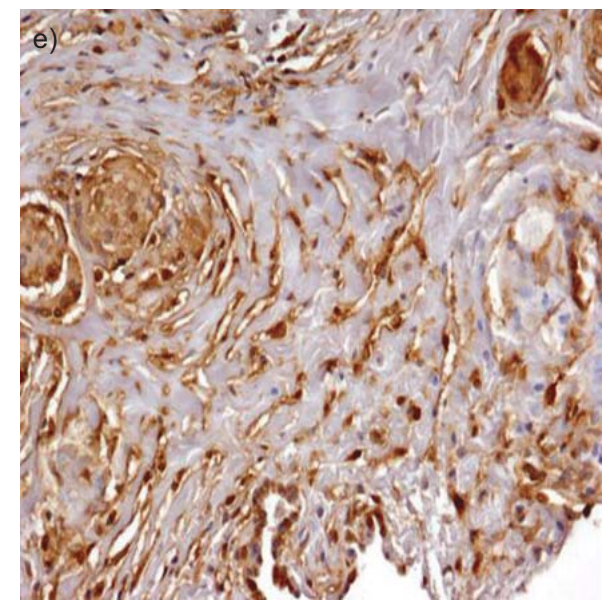

Anti-CXCL9

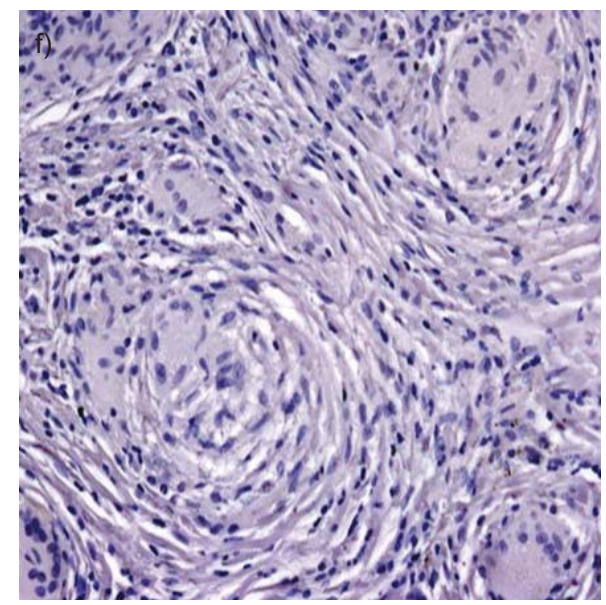

Control

FIGURE 5. Representative immunohistochemistry for a, b, $d$ and e) CXC chemokine ligand (CXCL)9 and c and f) isotype control antibody on lung biopsy tissue from a and d) stage I and b, c, e and f) stage II sarcoidosis. CXCL9 protein expression was found in epithelioid histiocytes and multinucleated giant cells forming non-necrotising granulomas, as well as from surrounding alveolar macrophages. Panels were photographed at $a-c) 50 \times$ and $d-f$ ) $200 \times$ original magnification.

attributable to our larger control group or the unlikely possibility that some of our healthy controls, despite a normal chest radiograph, the absence of clinical symptomatolgy or positive microbiological data, may have had an indolent infection not diagnosed at the time of bronchoscopy. Similar to our data, SugIYAMA et al. [10] did not find elevated levels of CXCL10 across the multiple stages of sarcoidosis. Based on these inconsistent studies and the heterogeneity of pulmonary sarcoidosis in terms of stage, immunosuppressive regimen and active versus inactive disease, future, appropriately powered, prospective studies will be necessary to determine whether there are significant elevations of CXCL10 in BALF from patients with pulmonary sarcoidosis.

While CXCL10 is an important IFN-inducible ELR CXC chemokine, there are two other members of this subfamily, CXCL9 and CXCL11, which share similar biological functions and interact with the same functional cell surface receptor, CXCR3. We found a nonsignificant trend towards elevated levels of CXCL11 from patients with pulmonary sarcoidosis. These elevations were predominately from stage I sarcoid patients. A recent study by ANTONIOU et al. [11] demonstrated lower levels of CXCL11 in BALF from patients with pulmonary sarcoidosis compared with controls. We ascribe these conflicting results to differences in the sarcoid populations. Specifically, the study by ANTONIOU et al. [11] included a total of 20 patients with pulmonary sarcoidosis, of which $25 \%$ had stage III disease (stage I $(n=8)$, stage II $(n=7)$ and stage III $(n=5))$. In contrast, our study involved only $12 \%$ of patients with stage III sarcoidosis. These differences in patient populations become critical as pathological investigations involving lung biopsies from patients with late-stage sarcoidosis usually demonstrate an end-stage fibrotic disease that can be absent of granulomas and can be essentially indistinguishable from usual interstitial pneumonitis (UIP) [24, 25]. Importantly, UIP lung biopsies have been shown to express a skewed type 2 immune response [26, 27]. Collectively, this suggests that early stages of sarcoidosis have an overwhelming type I granulomatous immune response that shifts to a profibrotic type 2 immune response during late-stage sarcoidosis. Based on this notion, we suspect that the study by ANTONIOU et al. [11], involving a larger percentage of patients with stage III 
Stage I
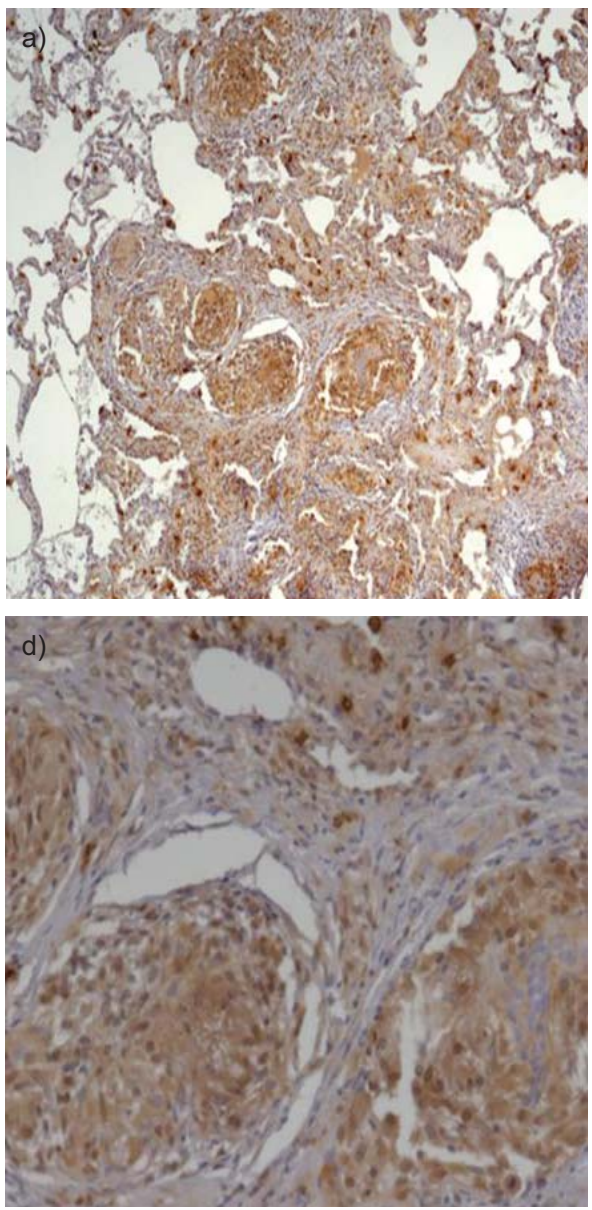

Anti-CXCR3
Stage II
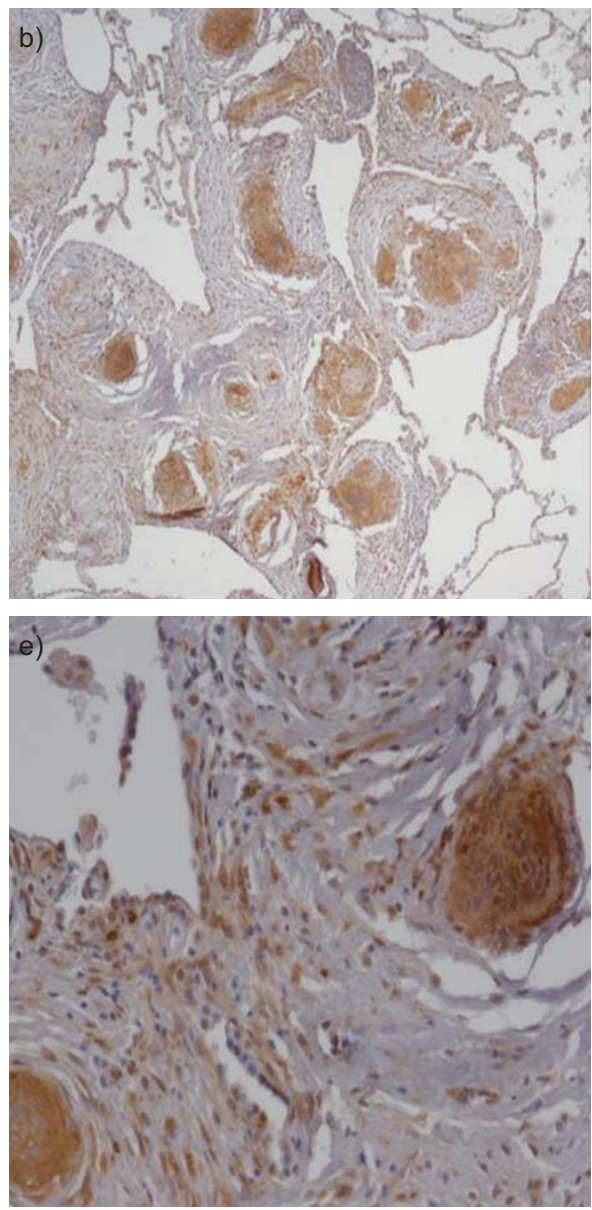

Anti-CXCR3
Stage II
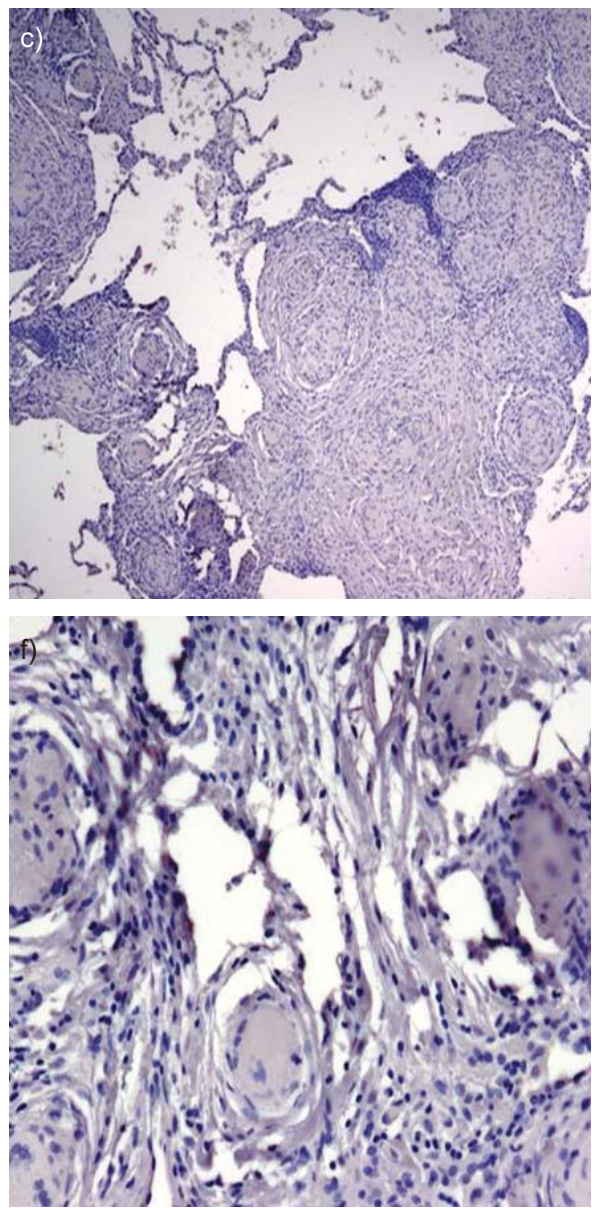

Control

FIGURE 6. Representative immunohistochemistry for a, b, d and e) CXC chemokine receptor (CXCR)3 and c and f) appropriate isotype control antibody on lung biopsy tissue from a and d) stage I and b, c, e and f) stage II sarcoidosis. CXCR3 protein expression was found in both epithelioid histiocytes and multinucleated giant cells within the granulomatous formations, as well as infiltrating interstitial mononuclear cells. Panels were photographed at a-c) $50 \times$ and d-f) $200 \times$ original magnification.

sarcoidosis and thus a larger proportion of patients with a predominant type 2 immune response, would be expected to demonstrate lower levels of CXCL11.

We found significantly elevated CXCL9 levels in patients with pulmonary sarcoidosis compared with controls. A post hoc analysis demonstrated that this was predominately due to both stage I and II disease. These results are supported by a smaller study, which also demonstrated significantly higher BALF levels of CXCL9 in patients with sarcoidosis $(n=44)$ compared with controls $(n=9)$ [12] and supports the notion that early stages of sarcoid have a biased type 1 immune response profile.

Interestingly, our investigation did not show a significant elevation of chemokine levels in sarcoid patients with alveolitis compared with those without alveolitis, although absolute median levels were higher for all three chemokines in the alveolitis groups. In addition, we did not find any correlation between chemokine levels and BALF lymphocyte counts (data not shown). This was surprising, as it has been shown that CXCR3 ligands are strong inducers of T-cell chemotaxis in pulmonary sarcoidosis [9]. To ensure the veracity of our findings and rule out the possibility of unappreciated chemokine protein degradation during storage of the BALF, we performed a correlation with chemokine protein levels and length of BALF cold storage time. We found no significant correlation in either our total sarcoid population or subgroups with and without alveolitis (data not shown). However, we see the same staining profiles of CXCL9 and CXCL11 by immunohistochemistry in patients with and without alveolitis. Based on this information, we assume that there is persistent chemokine protein expression from the granulomatous lesions in sarcoid patients both with and without alveolitis. This, in turn, leads to elevated chemokine levels in BALF of early-stage pulmonary sarcoidosis, irrespective of the presence or absence of alveolitis.

Finding elevated levels of CXCL11 in patients with stage I sarcoidosis and CXCL9 in stages I and II sarcoidosis led us to perform immunohistochemistry for these chemokines and their shared receptor CXCR3 on lung tissue, in order to determine their cellular sources. CXCL11 localised to epithelioid histiocytes and multinucleated giant cells forming non-necrotising 

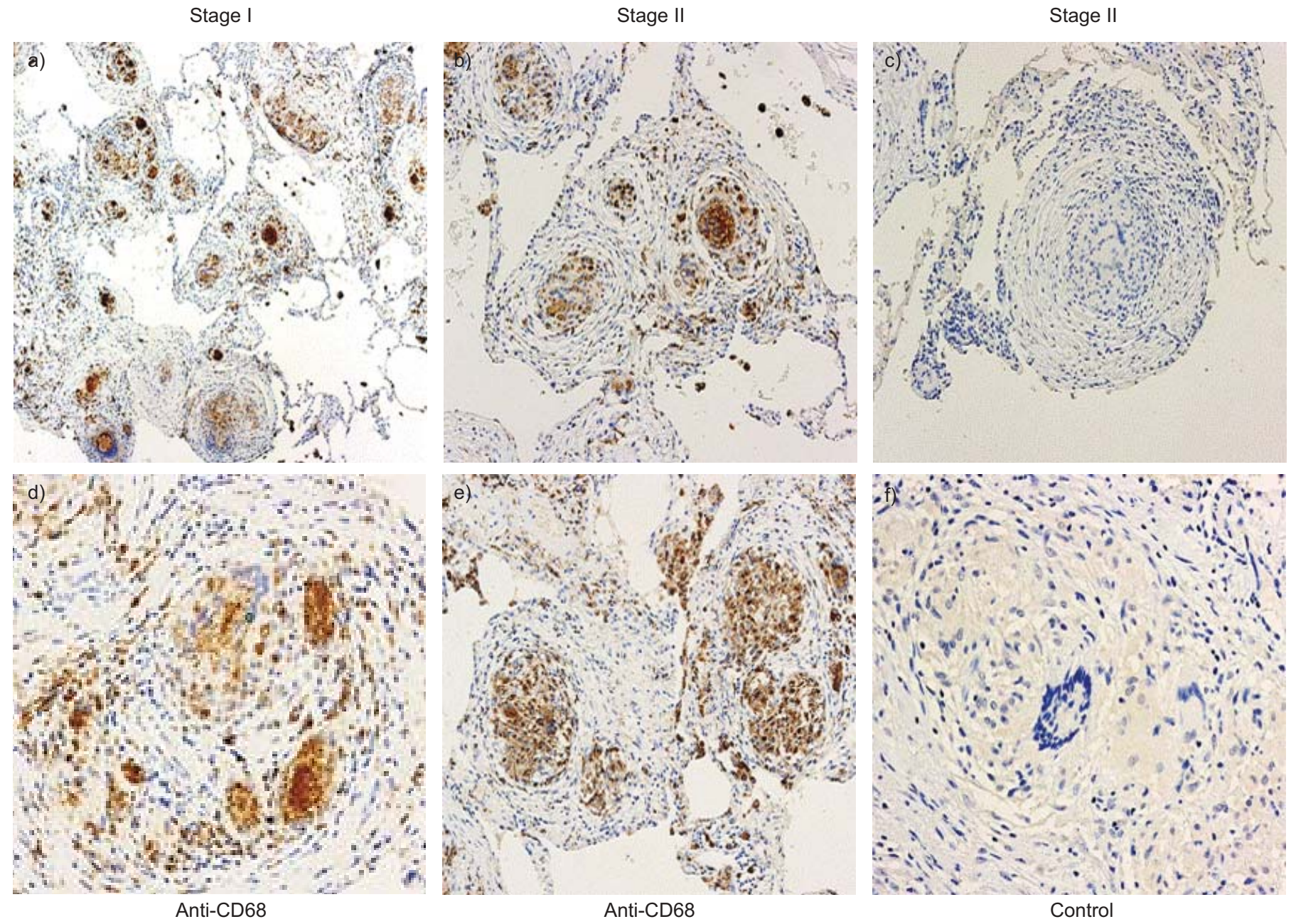

FIGURE 7. CXC chemokine receptor (CXCR)3 expression was confirmed in both epithelioid histiocytes and multinucleated giant cells using a, b, $d$ and e) the mononuclear phagocyte marker CD68 and c and f) appropriate isotype control antibody on lung biopsy tissue from a and d) stage I and b, c, e and f) stage II sarcoidosis. Panels were photographed at $a-c) 50 \times$ and $d-f$ ) $200 \times$ original magnification.

granulomas, as well as surrounding alveolar macrophages. CXCL9 also localised to epithelioid histiocytes within granulomas, but was less impressive than CXCL11 from multinucleated giant cells and surrounding alveolar macrophages, suggesting that specific cell populations are more responsible for the production of certain chemokines than others. These data are consistent with studies demonstrating that type I immune response cytokines, IL-12 and IL-18, are produced by epithelioid and multinucleated giant cells, which in turn upregulate the production of IFN- $\gamma$ from T-cells [28]. This then intimates the possibility that IFN- $\gamma$ stimulates mononuclear phagocytes to produce IFN-inducible ELR ${ }^{-}$CXC chemokines, which recruit more mononuclear cells expressing CXCR3, some of which also express IFN- $\gamma$, ultimately creating a positive feedback loop that eventually leads to granuloma formation (fig. 8).

We identified CXCR3 expression from T-cells infiltrating and surrounding the sarcoid granulomatous lesions. This is supported by studies finding CXCR3 expression on BAL lymphocytes from sarcoid patients [13]. Importantly, IL-2 has been found to be one of the most potent inducers of CXCR3 on lymphocytes [29], and multiple studies have suggested that IL-2 is pivotal in activating peripheral blood and BAL lymphocytes during pulmonary sarcoidosis [30-34]. Cooperatively, these results suggest that IL-2 is systemically priming T-cells to express CXCR3 while elevated CXCR3 ligands from the sarcoid lung are recruiting these lymphocytes, thereby perpetuating inflammation/granuloma formation (fig. 8). Recent studies have also suggested that macrophages akin to lymphocytes can be influenced by their inflammatory milieu. More specifically, similar to the T-helper cell (Th) type 1/Th2 lymphocyte paradigm (Th1 lymphocytes express IFN- $\gamma$, IL-12 and IL-18 as well as the cell receptors CCR5 and CXCR3, and Th2 lymphocytes express IL-4 and IL-13 as well as the cell surface receptors CCR3 and CCR4), macrophages can also polarise into type 1 (M1) or 2 (M2) macrophages [35-37]. Recently, microglia cells, the brain resident macrophages, have been found to express CXCR3 and contribute to specific neurological diseases [38-41]. We have expanded on these studies by demonstrating that lung cells of monocyte linage (e.g. epithelioid histiocytes and multinucleated giant cells) that form the pulmonary granulomas of sarcoidosis can express CXCR3. Taken together, 
a)

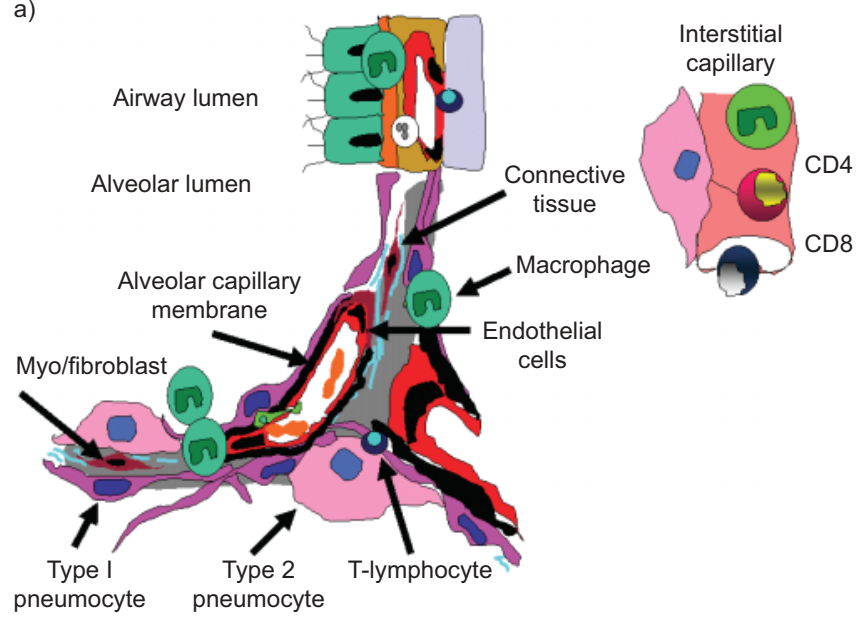

d)

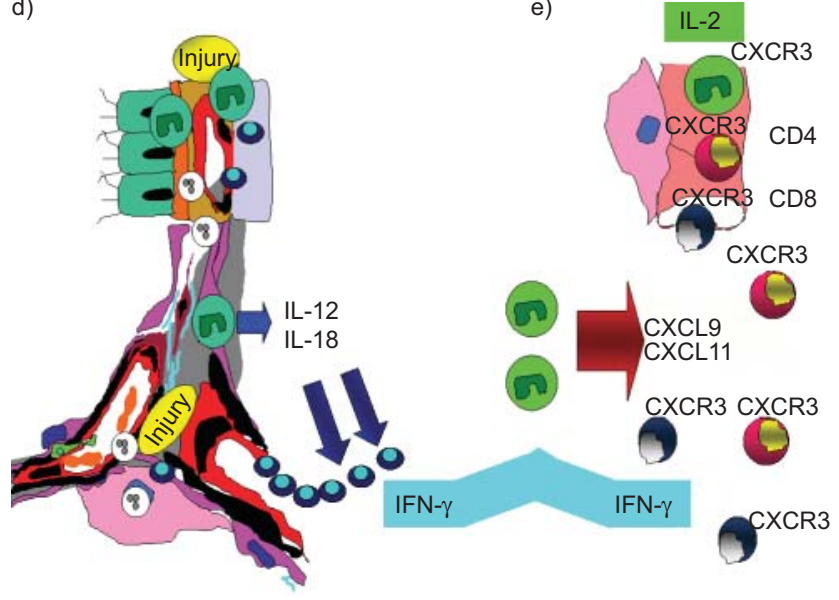

b)

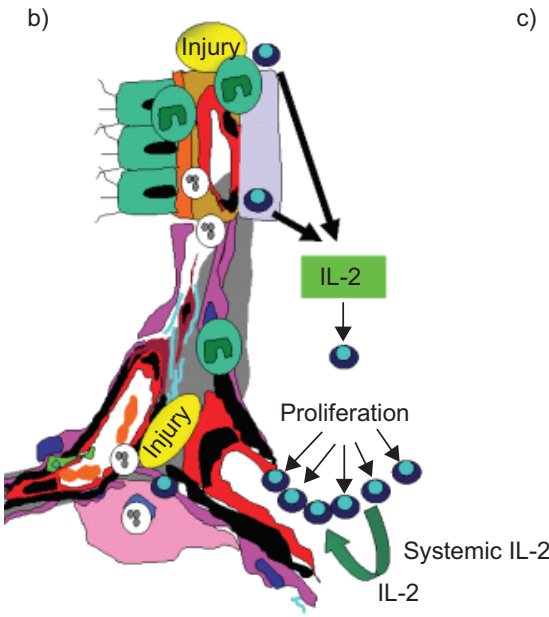

c)

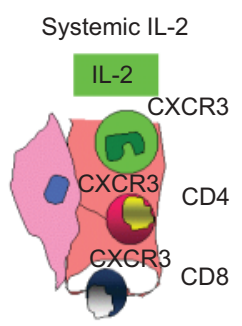

f)

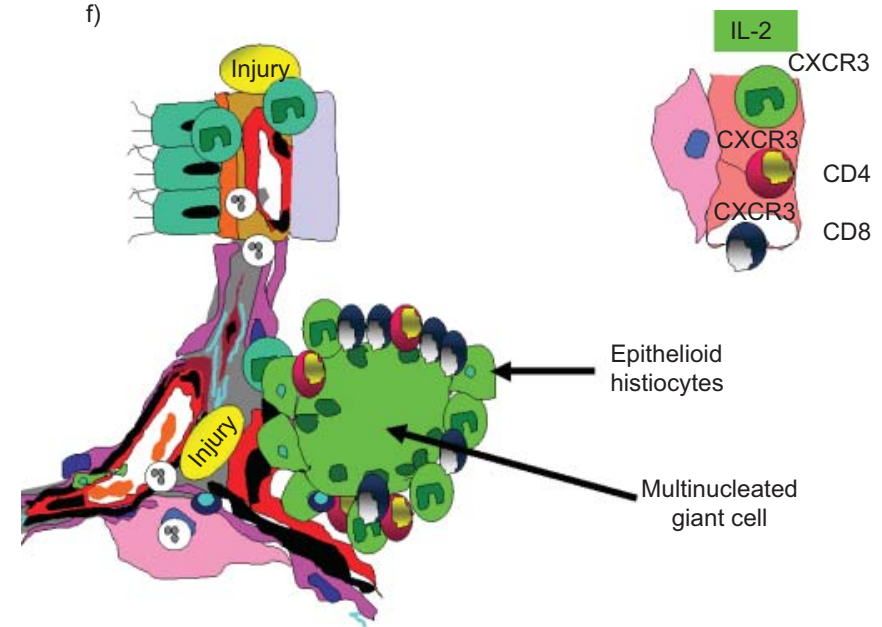

FIGURE 8. Suggested role of interferon (IFN)-inducible ELR- (lacking the sequence glutamic acid-leucine-arginine) CXC chemokines during the pathogenesis of pulmonary sarcoidosis. a) Normal airway leading to the alveolar-interstitial space. b) Unknown injury to the lower airway and alveolar space during pulmonary sarcoid leads to the expression of interleukin (IL)-2 from T-cells, causing T-cell proliferation. C) Systemic levels of IL-2 upregulate CXC chemokine receptor (CXCR)3 expression on mononuclear cells. d) Unknown injury triggers the release of IL-12 and IL-18 from mononuclear phagocytes, causing T-cells to release IFN- $\gamma$. e) IFN- $\gamma$ acts on mononuclear phagocytes, upregulating their expression of CXC chemokine ligand (CXCL)9 and CXCL11. In turn, these chemokines recruit more CXCR3-positive mononuclear cells, some of which express IFN- $\gamma$, creating a positive feedback loop. f) Ultimately these persistently recruited mononuclear cells create a type 1 immune response, allowing the coalescence of mononuclear phagocytes and epithelial histiocytes that eventually leads to pulmonary granuloma formation.

this suggests that the macrophages, epithelioid histocytes and multinucleated giant cells found in pulmonary sarcoid granulomas are of the M1 phenotype via their CXCR3 expression and, in part, are responsible for the type 1 immune response found during early stages of pulmonary sarcoidosis.

To our knowledge, this is the largest pulmonary sarcoidosis study involving IFN-inducible ELR ${ }^{-}$CXC chemokines in BALF. We have demonstrated an association between elevated BALF levels of CXCL9 and CXCL11 and pulmonary sarcoidosis and have shown that the cellular sources are predominately epithelioid histiocytes and multinucleated giant cells. Moreover, the sarcoid granulomas are made up of CXCR3expressing lymphocytes and cells of monocyte linage, such as the coalescence of epithioid histiocytes. These results suggest that during stage I and II sarcoidosis the CXCR3 ligands recruit CXCR3-expressing mononuclear cells, which propagate a type 1 immune response, ultimately causing granuloma formation.
Based on these studies, agents targeting the CXCR3/CXCR3 ligand biological axis may be avenues for future therapies in the treatment of sarcoidosis.

\section{SUPPORT STATEMENT}

This work was supported, in part, by grants from the National Institutes of Health (Bethesda, MD, USA; HL080206 to J.A. Belperio, HL060606 to R.M. Elashoff and HL66027 to R.M. Strieter) and a grant from Science Foundation Ireland (Dublin, Ireland) to M.P. Keane.

\section{STATEMENT OF INTEREST}

None declared.

\section{REFERENCES}

1 Boucek MM, Waltz DA, Edwards LB, et al. Registry of the International Society for Heart and Lung Transplantation: ninth official pediatric heart transplantation report - 2006. J Heart Lung Transplant 2006; 25: 893-903. 
2 Statement on sarcoidosis. Joint Statement of the American Thoracic Society (ATS), the European Respiratory Society (ERS) and the World Association of Sarcoidosis and Other Granulomatous Disorders (WASOG) adopted by the ATS Board of Directors and by the ERS Executive Committee, February 1999. Am J Respir Crit Care Med 1999; 160: 736-755.

3 Baughman RP, Teirstein AS, Judson MA, et al. Clinical characteristics of patients in a case control study of sarcoidosis. Am J Respir Crit Care Med 2001; 164: 1885-1889.

4 Newman LS, Rose CS, Maier LA. Sarcoidosis. N Engl J Med 1997; 336: 1224-1234.

5 Poletti V, Patelli M, Spiga L, et al. Transbronchial lung biopsy in pulmonary sarcoidosis. Is it an evaluable method in detection of disease activity? Chest 1986; 89: 361-365.

6 Belperio JA, Burdick MD, Keane MP, et al. The role of the CC chemokine, RANTES, in acute lung allograft rejection. J Immunol 2000; 165: 461-472.

7 Loetscher M, Gerber B, Loetscher P, et al. Chemokine receptor specific for IP10 and mig: structure, function, and expression in activated T-lymphocytes. J Exp Med 1996; 184: 963-969.

8 Keane MP, Strieter RM. The role of CXC chemokines in the regulation of angiogenesis. Chem Immunol 1999; 72: 86-101.

9 Agostini C, Cassatella M, Zambello R, et al. Involvement of the IP10 chemokine in sarcoid granulomatous reactions. J Immunol 1998; 161: 6413-6420.

10 Sugiyama K, Mukae H, Ishii $\mathrm{H}$, et al. Elevated levels of interferon $\gamma$-inducible protein-10 and epithelial neutrophil-activating peptide-78 in patients with pulmonary sarcoidosis. Respirology 2006; 11: 708-714.

11 Antoniou KM, Tzouvelekis A, Alexandrakis MG, et al. Different angiogenic activity in pulmonary sarcoidosis and idiopathic pulmonary fibrosis. Chest 2006; 130: 982-988.

12 Nishioka Y, Manabe K, Kishi J, et al. CXCL9 and 11 in patients with pulmonary sarcoidosis: a role of alveolar macrophages. Clin Exp Immunol 2007; 149: 317-326.

13 Agostini C, Facco M, Chilosi M, et al. Alveolar macrophage-T cell interactions during Th1-type sarcoid inflammation. Microsc Res Tech 2001; 53: 278-287.

14 Reich JM. Mortality of intrathoracic sarcoidosis in referral vs population-based settings: influence of stage, ethnicity, and corticosteroid therapy. Chest 2002; 121: 32-39.

15 Weigt SS, Elashoff RM, Keane MP, et al. Altered levels of CC chemokines during pulmonary CMV predict BOS and mortality post-lung transplantation. Am J Transplant 2008; 8: 1512-1522.

16 Belperio JA, Keane MP, Burdick MD, et al. Role of CXCR2/CXCR2 ligands in vascular remodeling during bronchiolitis obliterans syndrome. J Clin Invest 2005; 115: 1150-1162.

17 Belperio JA, DiGiovine B, Keane MP, et al. Interleukin-1 receptor antagonist as a biomarker for bronchiolitis obliterans syndrome in lung transplant recipients. Transplantation 2002; 73: 591-599.

18 Agostini C, Trentin L, Facco M, et al. Role of IL-15, IL-2, and their receptors in the development of $\mathrm{T}$ cell alveolitis in pulmonary sarcoidosis. J Immunol 1996; 157: 910-918.

19 Baughman RP, Strohofer SA, Buchsbaum J, et al. Release of tumor necrosis factor by alveolar macrophages of patients with sarcoidosis. J Lab Clin Med 1990; 115: 36-42.

20 Kreipe H, Radzun HJ, Heidorn K, et al. Proliferation, macrophage colony-stimulating factor, and macrophage colony-stimulating factor-receptor expression of alveolar macrophages in active sarcoidosis. Lab Invest 1990; 62: 697-703.
21 Moller DR, Forman JD, Liu MC, et al. Enhanced expression of IL-12 associated with Th1 cytokine profiles in active pulmonary sarcoidosis. J Immunol 1996; 156: 4952-4960.

22 Semenzato G, Adami F, Maschio N, et al. Immune mechanisms in interstitial lung diseases. Allergy 2000; 55: 1103-1120.

23 Baughman RP, Lower EE. The effect of corticosteroid or methotrexate therapy on lung lymphocytes and macrophages in sarcoidosis. Am Rev Respir Dis 1990; 142: 1268-1271.

24 Rosen Y. Pathology of sarcoidosis. Semin Respir Crit Care Med 2007; 28: 36-52.

25 Lynch JP 3rd, Ma YL, Koss MN, et al. Pulmonary sarcoidosis. Semin Respir Crit Care Med 2007; 28: 53-74.

26 Keane MP, Arenberg DA, Lynch JP 3rd, et al. The CXC chemokines, IL-8 and IP-10, regulate angiogenic activity in idiopathic pulmonary fibrosis. J Immunol 1997; 159: 1437-1443.

27 Meloni F, Caporali R, Marone Bianco A, et al. BAL cytokine profile in different interstitial lung diseases: a focus on systemic sclerosis. Sarcoidosis Vasc Diffuse Lung Dis 2004; 21: 111-118.

28 Shigehara K, Shijubo N, Ohmichi M, et al. IL-12 and IL-18 are increased and stimulate IFN- $\gamma$ production in sarcoid lungs. J Immunol 2001; 166: 642-649.

29 Beider K, Nagler A, Wald O, et al. Involvement of CXCR4 and IL-2 in the homing and retention of human NK and NK T cells to the bone marrow and spleen of NOD/SCID mice. Blood 2003; 102: 1951-1958.

30 Wahlstrom J, Katchar K, Wigzell H, et al. Analysis of intracellular cytokines in CD4+ and CD8+ lung and blood T cells in sarcoidosis. Am J Respir Crit Care Med 2001; 163: 115-121.

31 Keicho N, Kitamura K, Takaku F, et al. Serum concentration of soluble interleukin-2 receptor as a sensitive parameter of disease activity in sarcoidosis. Chest 1990; 98: 1125-1129.

32 Lawrence EC, Brousseau KP, Berger MB, et al. Elevated concentrations of soluble interleukin-2 receptors in serum samples and bronchoalveolar lavage fluids in active sarcoidosis. Am Rev Respir Dis 1988; 137: 759-764.

33 Grutters JC, Fellrath JM, Mulder L, et al. Serum soluble interleukin-2 receptor measurement in patients with sarcoidosis: a clinical evaluation. Chest 2003; 124: 186-195.

34 Prasse A, Georges CG, Biller H, et al. Th1 cytokine pattern in sarcoidosis is expressed by bronchoalveolar CD4+ and CD8+ T cells. Clin Exp Immunol 2000; 122: 241-248.

35 Mantovani A, Schioppa T, Porta C, et al. Role of tumor-associated macrophages in tumor progression and invasion. Cancer Metastasis Rev 2006; 25: 315-322.

36 Martinez FO, Sica A, Mantovani A, et al. Macrophage activation and polarization. Front Biosci 2008; 13: 453-461.

37 Sica A, Allavena P, Mantovani A. Cancer related inflammation: the macrophage connection. Cancer Lett 2008; 267: 204-215.

38 de Haas AH, Boddeke HW, Biber K. Region-specific expression of immunoregulatory proteins on microglia in the healthy CNS. Glia 2008; 56: 888-894.

39 de Jong EK, de Haas AH, Brouwer N, et al. Expression of CXCL4 in microglia in vitro and in vivo and its possible signaling through CXCR3. J Neurochem 2008; 105: 1726-1736.

40 Li H, Gang Z, Yuling H, et al. Different neurotropic pathogens elicit neurotoxic CCR9- or neurosupportive CXCR3-expressing microglia. J Immunol 2006; 177: 3644-3656.

41 Tanuma N, Sakuma H, Sasaki A, et al. Chemokine expression by astrocytes plays a role in microglia/macrophage activation and subsequent neurodegeneration in secondary progressive multiple sclerosis. Acta Neuropathol 2006; 112: 195-204. 